July 18, 2018

\title{
The Response to a Perturbation in the REFLECTION AMPLITUDE
}

\author{
Joshua Feinberg* \\ Department of Physics, \\ Oranim-University of Haifa, Tivon 36006, Israe*** \\ and \\ Department of Physics, \\ Technion - Israel Institute of Technology, Haifa 32000 Israel
}

\begin{abstract}
We apply inverse scattering theory to calculate the functional derivative of the potential $V(x)$ and wave function $\psi(x, k)$ of a one-dimensional Schrödinger operator with respect to the reflection amplitude $r(k)$.
\end{abstract}

Key Words: Schrödinger operator, inverse scattering theory, Gelfand-Levitan-Marchenko equation, soliton, SUSY QM.

PACS numbers: 02.30.Zz, 03.65.Db, 03.65.Nk, 02.30.Rz

*e-mail: joshua@physics.technion.ac.il

** permanent address 


\section{Introduction}

The computation of the effect of a perturbation in the potential $V(x)$ of a onedimensional Schrödinger operator

$$
H=-\partial_{x}^{2}+V(x)
$$

defined on the whole real line on the continuous part of the spectrum of $H$ is a standard (albeit non trivial) procedure in perturbation theory. For example, the response of the reflection amplitude $r(k)$ at momentum $k$ (in a setting where there is source at $x=+\infty)$ to an infinitesimal change in the potential is

$$
\frac{\delta r(k)}{\delta V(x)}=\frac{(t(k) \phi(x, k))^{2}}{2 i k} .
$$

The derivation of this result is given in the Appendix. Here $t(k)$ is the transmission amplitude and $t(k) \phi(x, k)$ is the solution of the Schrödinger equation

$$
\left(-\partial_{x}^{2}+V(x)-k^{2}\right) \Psi(x, k)=0
$$

which satisfies the scattering boundary conditions of this problem:

$$
\begin{aligned}
t(k) \phi(x, k) & =t(k) e^{-i k x}+o(1) & & x \rightarrow-\infty \\
t(k) \phi(x, k) & =\quad e^{-i k x}+r(k) e^{i k x}+o(1) & & x \rightarrow \infty .
\end{aligned}
$$

Obtaining the kernel inverse to (1.2), i.e., the response of the Schrödinger potential $V(x)$ (as well as the response of the wave function) to a change in the reflection amplitude $r(k)$, is a much more difficult problem, which we solve in this paper.

The explicit formulas we derive in this work express the local response of the potential and wavefunctions (see (3.13) and (3.15)) to a change in the reflection amplitude. Thus, our results add to the information which can be gleaned from the well-known trace identities of [1], the lowest of which reads

$$
\int_{-\infty}^{\infty} V(x) d x=-\frac{2}{\pi} \int_{0}^{\infty} \log \left[1-|r(k)|^{2}\right] d k-4 \sum_{l=1}^{N} \kappa_{l}
$$


where $E_{l}=-\kappa_{l}^{2}$ are the $N$ bound state energies (with $\kappa_{l}>0$ ), which tell us only about that response integrated over space.

A possibly interesting application of the results of this paper might be the investigation of perturbations around reflectionless potentials with arbitrary numbers of bound states[2], which play an important role in supersymmetric quantum mechanics[3] and the theory of solitons.

It would be useful at this point to introduce some additional notations and recall some basic facts, which will be used later on. For $k$ real, $H-k^{2}$ is real, and therefore $\Psi^{*}(x, k)=\Psi(x,-k)$ for any solution of (1.3). It follows that

$$
r^{*}(k)=r(-k) \quad \text { and } \quad t^{*}(k)=t(-k)
$$

in (1.4).

$\Psi(x, k)$ and $\Psi^{*}(x, k)$ are linearly independent solutions of (1.3), and the continuous spectrum is doubly degenerate at each $k^{2}>0$. In particular, $\phi(x, k)$ and $\phi^{*}(x, k)$ form a basis. Since they are degenerate in energy, their Wronskian is a non-zero constant. Equating its values at $x \rightarrow \pm \infty$, we obtain the probability conservation relation

$$
|r(k)|^{2}+|t(k)|^{2}=1 .
$$

An equally suitable basis is the pair of solutions $\psi(x, k)$ and $\psi^{*}(x, k)$ of $(1.3)$, in which $\psi(x, k)$ obeys the boundary condition

$$
\psi(x, k)=e^{-i k x}+o(1), \quad x \rightarrow \infty .
$$

Thus, $\psi^{*}(x, k)$ corresponds to a setting in which there is a source at $x=-\infty$.

We see from (1.4) and (1.8) that

$$
\phi(x, k) \underset{x \rightarrow \infty}{\longrightarrow} \frac{1}{t(k)} \psi(x, k)+\frac{r(k)}{t(k)} \psi^{*}(x, k) .
$$

This relation must hold for all $x$, since $\psi(x, k)$ and $\psi^{*}(x, k)$ form a basis everywhere. Adding to it the linear combination for $\phi^{*}(x, k)$, we may write the relation between the two bases as

$$
\left(\begin{array}{c}
\phi(x, k) \\
\phi^{*}(x, k)
\end{array}\right)=\left(\begin{array}{cc}
\frac{1}{t(k)} & \frac{r(k)}{t(k)} \\
\frac{r^{*}(k)}{t^{*}(k)} & \frac{1}{t^{*}(k)}
\end{array}\right)\left(\begin{array}{c}
\psi(x, k) \\
\psi^{*}(x, k)
\end{array}\right) .
$$


Note that the transformation matrix has a unit determinant. The inverse transformation is thus

$$
\left(\begin{array}{c}
\psi(x, k) \\
\psi^{*}(x, k)
\end{array}\right)=\left(\begin{array}{cc}
\frac{1}{t^{*}(k)} & -\frac{r(k)}{t(k)} \\
-\frac{r^{*}(k)}{t^{*}(k)} & \frac{1}{t(k)}
\end{array}\right)\left(\begin{array}{c}
\phi(x, k) \\
\phi^{*}(x, k)
\end{array}\right) .
$$

This paper is organized as follows: In the next section we present a lightning review of inverse scattering theory. In particular, we discuss the Gelfand-Levitan-Marchenko equation and its properties. We show that its solution is simply the boundary column of its resolvent kernel.

In section 3 we compute the variational derivative of the solution of the GelfandLevitan-Marchenko equation with respect to the reflection amplitude. Then we derive from it the corresponding derivatives of the potential and wavefunctions (Eqs. (3.13) and (3.15), respectively) in closed form.

In section 4 we demonstrate the consistency of our results by comparing their integrated form against known facts.

Finally, in the Appendix we provide some useful technical details. In particular, we present the derivation of (1.2), and also discuss briefly the case of reflectionless potentials.

\section{The Gelfand-Levitan-Marchenko Equation and Its Solution}

According to inverse scattering theory (IST) $[4,5,6,7]$, a Schrödinger operator (1.1) whose potential $V(x)$ tends asymptotically to zero fast enough, such that $\int_{-\infty}^{\infty}|V(x)|(1+$ $|x|) d x<\infty$, and thus supports only a finite number $N$ of bound states, is uniquely determined by the so-called scattering data. The scattering data are the reflection amplitude $r(k)$, and a finite set of $2 N$ real numbers

$$
\kappa_{1}>\kappa_{2}>\cdots \kappa_{N}>0 \quad \text { and } \quad c_{1}, c_{2}, \cdots c_{N}
$$

where $E_{l}=-\kappa_{l}^{2}$ is the $l$ th bound state energy, and where $c_{l}$ appears in the asymptotic behavior of the $l$ th normalized bound state wave function as $c_{l} \exp -\kappa_{l}|x|$ (and thus 
determines its "center of gravity").

The prescribed reflection amplitude $r(k)$ can be taken as any complex-valued function which satisfies (for $k$ real):

$$
\begin{aligned}
r(-k) & =r^{*}(k) & & \\
|r(k)| & <1, & & k \neq 0 \quad \text { and } \\
r(k) & =O\left(\frac{1}{k}\right), & & |k| \rightarrow \infty .
\end{aligned}
$$

The first two conditions were already mentioned in (1.6) and (1.7), and the third one reflects the fact that $V(x)$ is a small perturbation at high energy. In addition to these conditions, there is a less obvious technical condition that the Fourier transform $B(x)=\int_{-\infty}^{\infty} \frac{r(k)}{t(k)} e^{i k x} d k$, should satisfy the bound $\int_{-\infty}^{\infty}(1+|x|)\left|\frac{d B(x)}{d x}\right| d x<\infty$. Note further that $t(k)$ is completely determined by $r(k)$ as

$$
\frac{t(k)}{\sqrt{1-|r(k)|^{2}}}=\left(\prod_{l=1}^{N} \frac{k+i \kappa_{l}}{k-i \kappa_{l}}\right) \exp \left(\frac{1}{2 \pi i} \text { P.P. } \int_{-\infty}^{\infty} \frac{\log \left[1-|r(q)|^{2}\right]}{q-k} d q\right) \text {. }
$$

Given the scattering data, IST instructs us to determine a certain real transformation kernel $K(x, y)$, bounded on the domain $y \geq x$, which maps the wave functions of the free Schrödinger operator $H_{0}=-\partial_{x}^{2}$ onto those of the operator $H$ in (1.1). For example, the left moving wave $e^{-i k x}$ is mapped onto

$$
\psi(x, k)=e^{-i k x}+\int_{x}^{\infty} K(x, y) e^{-i k y} d y
$$

which is evidently the solution of (1.3) satisfying the boundary condition (1.8) mentioned above. Finally, the potential $V(x)$ in (1.1) is determined by $K(x, y)$ according to

$$
V(x)=-2 \frac{d}{d x} K(x, x) .
$$

The kernel $K(x, y)$ is determined as the solution of the Gelfand-Levitan-Marchenko equation (GLM) $[4,5,6,7]$,

$$
K(x, y)+F(x+y)+\int_{x}^{\infty} K(x, z) F(z+y) d z=0
$$


where the real function $F(x)$ is

$$
F(x)=\sum_{l=1}^{N} c_{l}^{2} e^{-\kappa_{l} x}+\frac{1}{2 \pi} \int_{-\infty}^{\infty} r(k) e^{i k x} d k .
$$

For fixed $x,(2.6)$ is a Fredholm integral equation of the second type in the unknown function $\Phi(y)=K(x, y)$,

$$
\Phi(y)=f(y)+\lambda \int_{x}^{\infty} N(y, z) \Phi(z),
$$

with symmetric real kernel and given function

$$
N(y, z)=N(z, y)=-F(y+z), \quad f(y)=-F(x+y)=N(y, x)
$$

respectively, and spectral parameter $\lambda=1$.

It is known that (2.6) has a unique solution, i.e., the Fredholm determinant of (2.8) is not null at $\lambda=1[5,6]$. It is easy to demonstrate this property in the case of reflectionless potentials (for which $r(k)=0$ for all $k$ ), as we show in the Appendix.

The unique solution of (2.8) at $\lambda=1$ is given by

$$
\Phi(y)=f(y)+\int_{x}^{\infty} R(y, z ; 1) f(z) d z,
$$

where $R(y, z ; \lambda)$ is the resolvent kernel of (2.8). Note that for real values of $\lambda$, $R(y, z ; \lambda)$ is manifestly real, when it exists.

It is useful at this point to introduce the operator $\hat{N}$ and the vectors $|\Phi\rangle$ and $|f\rangle$, which correspond to the kernel $N(y, z)$ and functions $\Phi(y)$ and $f(y)$. Thus, in obvious notations, $N(y, z)=\langle y|\hat{N}| z\rangle=-F(y+z),\langle y \mid f\rangle=f(y)=\langle y|\hat{N}| x\rangle$ and $\Phi(y)=\langle y \mid \Phi\rangle$. Then, it is easy to see from (2.8) that

$$
|\Phi\rangle=\frac{1}{1-\hat{N}}|f\rangle=\frac{\hat{N}}{1-\hat{N}}|x\rangle .
$$

Similarly, from (2.10) we deduce that $R(y, z ; 1)=\langle y|\hat{R}| z\rangle$, where

$$
\hat{R}=\frac{1}{1-\hat{N}}-\mathbf{1}=\frac{\hat{N}}{1-\hat{N}} .
$$


Thus, by comparing (2.11) and (2.12), we conclude that $|\Phi\rangle=\hat{R}|x\rangle$, i.e.,

$$
\Phi(y)=K(x, y)=\langle y|\hat{R}| x\rangle
$$

The solution of the GLM equation (2.6) coincides with the $x$ th column of its resolvent kernel. It is manifestly a real function of $x$ and $y$.

\section{The Variational Derivatives}

In view of (2.13), it is straightforward to compute the variation of $K(x, y)$ under small perturbations in $\hat{N}$. Thus, consider a perturbation $\hat{N} \rightarrow \hat{N}+\delta \hat{N}$, which induces the variation $\hat{R} \rightarrow \hat{R}+\frac{1}{\mathbf{1}-\hat{N}} \delta \hat{N} \frac{1}{\mathbf{1 - N}}$. Consequently $\frac{\delta R(y, z ; 1)}{\delta N(a, b)}=\langle y|(\mathbf{1}+\hat{R})| a\rangle\langle b|(\mathbf{1}+\hat{R})| z\rangle$. Thus, from (2.13)

$$
\frac{\delta K(x, y)}{\delta N(a, b)}=(\delta(y-a)+R(y, a ; 1))(\delta(b-x)+K(x, b)) .
$$

In this work we are interested in variations $\delta \hat{N}$ which result from a change $\delta r(k)$ in the reflection amplitude. Due to the first condition in $(2.2)$ we must impose $\delta r(-k)=$ $\delta r^{*}(k)$. Thus, with no loss of generality, we take the positive components $r(k), k \geq 0$ as the independent functional variables. Keeping that in mind, we obtain from (2.7) and (2.9) that

$$
\frac{\delta N(a, b)}{\delta r(k)}=-\frac{1}{2 \pi} e^{i k(a+b)} .
$$

Thus, from (3.1) and (3.2) we obtain

$$
\begin{aligned}
& \frac{\delta K(x, y)}{\delta r(k)}=\int_{x}^{\infty} \frac{\delta K(x, y)}{\delta N(a, b)} \frac{\delta N(a, b)}{\delta r(k)} d a d b= \\
& -\frac{1}{2 \pi}\left(e^{i k y}+\int_{x}^{\infty} R(y, a ; 1) e^{i k a} d a\right)\left(e^{i k x}+\int_{x}^{\infty} K(x, b) e^{i k b} d b\right) .
\end{aligned}
$$

From (2.4) and from the reality of $K(x, y)$ and $k$, we recognize the last factor in (3.3) simply as $\psi^{*}(x, k)$. Thus,

$$
\frac{\delta K(x, y)}{\delta r(k)}=-\frac{1}{2 \pi}\left(e^{i k y}+\int_{x}^{\infty} R(y, a ; 1) e^{i k a} d a\right) \psi^{*}(x, k)
$$


In order to simplify (3.4) further, we have to study the function

$$
\omega(y, x ; k)=\int_{x}^{\infty} R(y, a ; 1) e^{i k a} d a .
$$

Observe from (2.10) (considered with a generic given function $f(y))$, that $\Omega(y, x ; k)=$ $e^{i k y}+\omega(y, x ; k)$ is the unique solution of the Fredholm equation $\Omega(y, x ; k)=e^{i k y}+$ $\int_{x}^{\infty} N(y, z) \Omega(z, x ; k) d z$, from which we infer that $\omega(y, x ; k)$ is the unique solution of

$$
\omega(y, x ; k)=-G(y, x ; k)+\int_{x}^{\infty} N(y, z) \omega(z, x ; k) d z
$$

where

$$
G(y, x ; k)=\int_{x}^{\infty} F(y+z) e^{i k z} d z .
$$

Thus, $\partial_{x} \omega(y, x ; k)$ satisfies

$$
\frac{\partial \omega(y, x ; k)}{\partial x}=F(x+y)\left(e^{i k x}+\omega(x, x ; k)\right)+\int_{x}^{\infty} N(y, z) \frac{\partial \omega(z, x ; k)}{\partial x} d z .
$$

From $(3.5),(2.13)$ and the fact that $R(y, z ; 1)=R(z, y ; 1)$, we obtain that $\omega(x, x ; k)=$ $\int_{x}^{\infty} R(a, x ; 1) e^{i k a} d a=\int_{x}^{\infty} K(x, a) e^{i k a} d a$. Thus, the inhomogeneous term in (3.8) is simply $F(x+y)\left(e^{i k x}+\int_{x}^{\infty} K(x, a) e^{i k a} d a\right)=F(x+y) \psi^{*}(x, k)=-f(y) \psi^{*}(x, k)$, where we used (2.4). It is the given function $f(y)$ in (2.8) multiplied by a $y$-independent factor $-\psi^{*}(x, k)$. Thus, from linearity, the unique solution of $(3.8)$ is simply the solution of (2.8), multiplied by the same factor, namely,

$$
\frac{\partial \omega(y, x ; k)}{\partial x}=-K(x, y) \psi^{*}(x, k) \text {. }
$$

The initial condition for this equation at $x=y$ is obviously $\omega(y, y ; k)=\int_{y}^{\infty} K(y, a) e^{i k a} d a=$ $\psi^{*}(y, k)-e^{i k y}$. Thus,

$$
\omega(y, x ; k)=\int_{x}^{y} \psi^{*}(z, k) K(z, y) d z+\psi^{*}(y, k)-e^{i k y} .
$$

Substituting this result into (3.4) we obtain our first main result:

$$
\frac{\delta K(x, y)}{\delta r(k)}=-\frac{1}{2 \pi}\left(\psi^{*}(y, k)+\int_{x}^{y} \psi^{*}(z, k) K(z, y) d z\right) \psi^{*}(x, k) .
$$


Since $K(x, y)$ is a real kernel, we can write (3.11) alternatively as

$$
\frac{\delta K(x, y)}{\delta r^{*}(k)}=-\frac{1}{2 \pi}\left(\psi(y, k)+\int_{x}^{y} \psi(z, k) K(z, y) d z\right) \psi(x, k) .
$$

The formula for $\frac{\delta K(x, y)}{\delta r^{*}(k)}$ is the key for obtaining the functional derivatives of the wave function $\psi(x, k)$ and potential $V(x)$ with respect to the reflection amplitude $r(k)$, since the former are linear in $K(x, y)$. Thus, from (2.5) and (3.12) we obtain our second main result:

$$
\frac{\delta V(x)}{\delta r^{*}(k)}=-2 \frac{d}{d x} \frac{\delta K(x, x)}{\delta r^{*}(k)}=\frac{1}{\pi} \frac{d}{d x} \psi^{2}(x, k) .
$$

Similarly, from (2.4) and (3.12) we obtain

$$
\frac{\delta \psi(x, k)}{\delta r^{*}(q)}=-\frac{1}{2 \pi}\left[\int_{x}^{\infty} e^{-i k y}\left(\psi(y, q)+\int_{x}^{y} \psi(z, q) K(z, y) d z\right) d y\right] \psi(x, q) .
$$

Reversing the order of integrations in the second integral according to

$$
\int_{x}^{\infty} e^{-i k y}\left(\int_{x}^{y} \psi(z, q) K(z, y) d z\right) d y=\int_{x}^{\infty} \psi(z, q)\left(\int_{z}^{\infty} e^{-i k y} K(z, y) d y\right) d z
$$

and recognizing the $y$-integral on the right hand side of the last equation as $\int_{z}^{\infty} e^{-i k y} K(z, y) d y=\psi(z, k)-e^{-i k z}$, we obtain from (3.14) our third main result:

$$
\frac{\delta \psi(x, k)}{\delta r^{*}(q)}=-\frac{1}{2 \pi}\left(\int_{x}^{\infty} \psi(z, q) \psi(z, k) d z\right) \psi(x, q)
$$

It is interesting to note that both (3.13) and (3.15) are expressed purely in terms of the wave function $\psi(x, k)$. Note, however, that (3.13) is local in $\psi$, whereas (3.15) is highly nonlocal. It would be interesting to interpret these features from a physical point of view.

\section{Consistency Checks of (3.13) and (3.15)}

The integrated form of (3.13) should agree with the derivative $\frac{\delta}{\delta r^{*}(k)} \int_{-\infty}^{\infty} V(x) d x$ obtained from the trace identity (1.5). Note that (1.5) is expressed purely in terms 
of the positive Fourier modes of $r(k)$, the independent functional variables in our problem. Thus, taking the derivative of (1.5), we obtain

$$
\frac{\delta}{\delta r^{*}(k)} \int_{-\infty}^{\infty} V(x) d x=-\frac{2}{\pi} \frac{\delta}{\delta r^{*}(k)} \int_{0}^{\infty} \log \left[1-|r(q)|^{2}\right] d q=\frac{2}{\pi} \frac{r(k)}{|t(k)|^{2}},
$$

where we used $|t(k)|^{2}=1-|r(k)|^{2}$. This result should be confronted with the integrated form of (3.13). We obtain from the latter

$$
\frac{\delta}{\delta r^{*}(k)} \int_{-\infty}^{\infty} V(x) d x=\frac{1}{\pi} \quad \lim _{L \rightarrow \infty}\left[\psi^{2}(x, k)\right]_{-L}^{L}
$$

From (1.8) we observe that $\psi(L, k) \simeq e^{-i k L}$. Similarly, from (1.10) and (1.4) we deduce the asymptotic behavior

$$
\psi(-L, k) \simeq \frac{1}{t^{*}(k)} e^{i k L}-\frac{r(k)}{t(k)} e^{-i k L} .
$$

Substituting (4.3) and $\psi(L, k) \simeq e^{-i k L}$ in (4.2), we obtain

$$
\frac{\delta}{\delta r^{*}(k)} \int_{-\infty}^{\infty} V(x) d x=\frac{1}{\pi} \lim _{L \rightarrow \infty}\left[e^{-2 i k L}\left(1-\frac{r^{2}}{t^{2}}\right)-\frac{1}{\left(t^{*}\right)^{2}} e^{2 i k L}+\frac{2 r}{|t|^{2}}\right]
$$

which coincides in the limit with (4.1) in the sense of distributions, since the first two rapidly oscillating terms on the right hand side of (4.4), when smeared against any continuous bounded test function $U(k)$ will integrate to zero in the limit $L \rightarrow \infty$, due to the Riemann-Lebesgue lemma. Thus, (3.13) has passed its first test.

A less trivial test of (3.13) arises from comparing the complex conjugate equation of (3.13), $\frac{\delta V(x)}{\delta r(k)}=\frac{1}{\pi} \frac{d}{d x} \psi^{* 2}(x, k)$ and (1.2). We see that one kernel is the inverse of the other: $\int_{-\infty}^{\infty} \frac{\delta r(k)}{\delta V(x)} \frac{\delta V(x)}{\delta r(q)} d x=\delta(k-q)$. Thus, we must verify that

$$
\Gamma(k, q)=\frac{t^{2}(k)}{2 \pi i k} \int_{-\infty}^{\infty} \phi^{2}(x, k) \frac{d}{d x} \psi^{* 2}(x, q) d x
$$

is equal to $\delta(k-q)$. Using the identity

$$
\int_{-\infty}^{\infty} F^{2} \frac{d}{d x} G^{2} d x=\frac{1}{2}\left[(F G)^{2}\right]_{-\infty}^{\infty}+\int_{-\infty}^{\infty} F G\left(F \partial_{x} G-G \partial_{x} F\right) d x
$$


with $F=\phi(x, k)$ and $G=\psi^{*}(x, q)$, we write $(4.5)$ as

$$
\Gamma(k, q)=\frac{t^{2}(k)}{2 \pi i k}\left\{\frac{1}{2}\left[\left(\phi(x, k) \psi^{*}(x, q)\right)^{2}\right]_{-\infty}^{\infty}+\int_{-\infty}^{\infty} \phi(x, k) \psi^{*}(x, k) W(x, k, q) d x\right\}
$$

where

$$
W(x, k, q)=\phi(x, k) \partial_{x} \psi^{*}(x, q)-\psi^{*}(x, q) \partial_{x} \phi(x, k)
$$

is the Wronskian of $\phi(x, k)$ and $\psi^{*}(x, q)$. Next, from the Schrödinger equation for these two functions it is easy to obtain the relation

$$
\phi(x, k) \psi^{*}(x, q)=\frac{1}{k^{2}-q^{2}} \partial_{x} W(x, k, q) .
$$

Substituting the last equation in (4.6) we see that the integral is given entirely by boundary terms as

$$
\Gamma(k, q)=\frac{t^{2}(k)}{4 \pi i k}\left[\left(\phi(x, k) \psi^{*}(x, q)\right)^{2}+\frac{1}{k^{2}-q^{2}} W^{2}(x, k, q)\right]_{-\infty}^{\infty} .
$$

In order to proceed in the clearest and simplest way, we shall make a few observations. First, observe from (1.4), (1.8) and (4.3) that the difference $\left(\phi(L, k) \psi^{*}(L, q)\right)^{2}-$ $\left(\phi(-L, k) \psi^{*}(-L, q)\right)^{2}$ is rapidly oscillating in the limit $L \rightarrow \infty$ for all $k, q$. Thus, as a distribution acting on bounded continuous functions of $k$ and $q$ it tends to zero. Thus, we should focus on the second term. When $k^{2}-q^{2} \neq 0$, the difference $W^{2}(L, k, q)-$ $W^{2}(-L, k, q)$ oscillates rapidly, and tends to zero, in the sense of distributions, in the limit $L \rightarrow \infty$, similarly to the first term. Recall that for $k^{2}=q^{2}$, i.e., when $\phi(x, k)$ and $\psi^{*}(x, q)$ correspond to the same energy, $W(x, k, q)$ is a constant, and thus cannot oscillate. In that case, however, the denominator in front of this term vanishes. Thus we should study the limit $q^{2} \rightarrow k^{2}$ carefully.

Let us concentrate then on the region $k^{2} \simeq q^{2}$. Since we have taken the positive Fourier modes of $r(k)$ as the independent functional variables, it is enough to study the case $q \rightarrow k$. Thus, assuming $k-q=\epsilon$ with $\left|\frac{\epsilon}{k}\right|<<1$ and setting $k+q \simeq 2 k$, we obtain from (1.4), (1.8) and (4.3) that

$$
\begin{aligned}
W(L, k, k-\epsilon) & \simeq \frac{i}{t(k)}\left(2 k e^{-i \epsilon L}-\epsilon r(k) e^{2 i k L}\right) \\
W(-L, k, k-\epsilon) & \simeq \frac{i}{t(k)}\left(2 k e^{i \epsilon L}-\epsilon t(k) \frac{r^{*}(k)}{t^{*}(k)} e^{2 i k L}\right) .
\end{aligned}
$$


Substituting (4.10) and $k^{2}-q^{2} \simeq 2 k \epsilon$ in (4.9), and recalling the first observation made right below (4.9), we finally obtain

$$
\Gamma(k, q)=\frac{\sin (2 \epsilon L)}{\pi \epsilon}+\text { R.O.T. }
$$

where the acronym R.O.T. stands for the rapidly oscillating terms that tend to zero in the sense of distributions. The first term, tends of course to $\delta(k-q)$ as $L \rightarrow \infty$, as desired. (3.13) has passed the second check!

Our last consistency check concerns (3.15). The point is that for $x \rightarrow-\infty$, the integral on the right hand side of (3.15) is the orthogonality relation

$$
\int_{-\infty}^{\infty} \psi(z, q) \psi(z, k) d z=\frac{2 \pi}{|t(k)|^{2}}(\delta(k+q)-r(k) \delta(k-q)) .
$$

Let us sketch the proof of (4.12): in analogy with (4.8) we deduce that

$$
\psi(x, k) \psi(x, q)=\frac{1}{k^{2}-q^{2}} \partial_{x} \tilde{W}(x, k, q)
$$

where

$$
\tilde{W}(x, k, q)=\psi(x, k) \partial_{x} \psi(x, q)-\psi(x, q) \partial_{x} \psi(x, k)
$$

is the Wronskian of the functions involved. Then, after integration, we obtain

$$
\int_{-L}^{L} \psi(z, q) \psi(z, k) d z=\frac{1}{k^{2}-q^{2}}[\tilde{W}(L, k, q)-\tilde{W}(-L, k, q)]
$$

and consider this result as $L \rightarrow \infty$, using the asymptotic behavior (1.8) and (4.3). For generic values of $k, q$, the difference of Wronskians is a rapidly oscillating function, which as a distribution, tends to zero as $L \rightarrow \infty$. As in the previous discussion, we observe that when $k^{2}=q^{2}, \tilde{W}(x, k, q)$ is independent of $x$, and thus does not oscillate. By studying the limit $q \rightarrow \pm k$ carefully, we deduce (4.12).

On the left hand side of (3.15) we have

$$
\frac{\delta \psi(-L, k)}{\delta r(-q)}=\frac{\delta}{\delta r(-q)}\left(\frac{1}{t(-k)} e^{i k L}-\frac{r(k)}{t(k)} e^{-i k L}\right)
$$


where we used (4.3). Then, from (2.3) it follows that

$$
\begin{aligned}
\frac{\delta t^{-1}(k)}{\delta r(p)} & =\frac{1}{2 t(k)} \frac{r^{*}(p)}{|t(p)|^{2}}\left[\delta(p-k)+\delta(p+k)+\frac{1}{i \pi} \mathrm{P} . \mathrm{P} \cdot\left(\frac{1}{p-k}-\frac{1}{p+k}\right)\right] \\
& =\frac{1}{2 \pi i t(k)} \frac{r^{*}(p)}{|t(p)|^{2}}\left(\frac{1}{p-k-i \epsilon}-\frac{1}{p+k+i \epsilon}\right) .
\end{aligned}
$$

Applying (4.16) to (4.15), and studying the resulting expression around $k \simeq q>0$,

we find (keeping only the singular terms) $\frac{1}{2 \pi \epsilon} \frac{r(k)}{|t(k)|^{2}} \psi(-L, k)$, in accordance with the right hand side of (3.15) and (4.12).

\section{Appendix: Miscellaneous Technical Details}

\section{A.1 Perturbation Theory in the Continuum: Proof of (1.2)}

Consider a perturbation $\delta V(x)$ of the Schrödinger equation (1.3). To make the problem well-defined, we assume that $\delta V(x)$ is localized around some point $x_{0}$.

Under this perturbation, the solution of (1.3) will be shifted $\Psi(x) \rightarrow \Psi(x)+\delta \Psi(x)$, and our task is to compute $\delta \Psi(x)$ to first order in $\delta V(x)$. To this order, we have to solve the equation

$$
\left(H-k^{2}\right) \delta \Psi(x, k)=-\delta V(x) \Psi(x, k)
$$

The general solution is given in terms of the Green's function $G(x, y ; k)$ of the operator $H-k^{2}$ (defined with the appropriate boundary conditions) as

$$
\delta \Psi(x, k)=-\int_{-\infty}^{\infty} G(x, y ; k) \delta V(y) \Psi(y, k) d y .
$$

Since this expression vanishes for $\delta V(y)=0$, there is no term which is a solution of the homogeneous equation.

The proper Green's function is the one which decays exponentially whenever one of its coordinate arguments tends to $\pm \infty$, when $k$ is lifted to the upper half complex plane. There is a unique such Green's function:

$$
G(x, y ; k)=\frac{1}{w(k)}\left[\theta(x-y) \psi^{*}(x, k) \phi(y, k)+\theta(y-x) \psi^{*}(y, k) \phi(x, k)\right]
$$


where

$$
w(k)=-W(x, k, k)=\frac{2 k}{i t(k)}
$$

from (4.7). Thus, in particular, for $\Psi(x, k)=\phi(x, k)$ we obtain

$$
\delta \phi(x, k)=-\frac{1}{w}\left[\psi^{*}(x, k) \int_{-\infty}^{x} \phi(y, k) \delta V(y) \phi(y, k) d y+\phi(x, k) \int_{x}^{\infty} \psi^{*}(y, k) \delta V(y) \phi(y, k) d y\right] .
$$

In the limit $x \rightarrow-\infty$, we see that

$$
\delta \phi(x, k) \rightarrow-\frac{\phi(x, k)}{w} \int_{-\infty}^{\infty} \psi^{*}(y, k) \delta V(y) \phi(y, k) d y
$$

Thus, the function

$$
\tilde{\phi}(x, k)=\frac{\phi(x, k)+\delta \phi(x, k)}{1-\frac{1}{w} \int_{-\infty}^{\infty} \psi^{*}(y, k) \delta V(y) \phi(y, k) d y}
$$

tends to $e^{-i k x}$ as $x \rightarrow-\infty$, and should be identified with the " $\phi$-function" (1.4) of the perturbed potential $V(x)+\delta V(x)$. In (A.7) we should keep, of course, only terms up to linear order in $\delta V(x)$. As $x \rightarrow \infty$, we see from (A.5), (1.4) and (1.8) that $\tilde{\phi}(x, k) \rightarrow \frac{1}{\tilde{t}(k)} e^{-i k x}+\frac{\tilde{r}(k)}{\tilde{t}(k)} e^{i k x}$, where $\tilde{t}(k)$ and $\tilde{r}(k)$ are the new scattering amplitudes, given by

$$
\begin{aligned}
& \frac{1}{\tilde{t}(k)}=\frac{1}{t(k)}\left[1+\frac{1}{w} \int_{-\infty}^{\infty} \psi^{*}(y, k) \delta V(y) \phi(y, k) d y\right] \\
& \frac{\tilde{r}(k)}{\tilde{t}(k)}=\frac{r(k)}{t(k)}\left[1+\frac{1}{w} \int_{-\infty}^{\infty}\left(\psi^{*}(y, k)-\frac{t(k)}{r(k)} \phi(y, k)\right) \delta V(y) \phi(y, k) d y\right]
\end{aligned}
$$

from which we infer, after some simple algebra, involving (1.9) that

$$
\delta r(k)=\tilde{r}(k)-r(k)=\frac{1}{2 i k} \int_{-\infty}^{\infty}(t(k) \phi(y, k))^{2} \delta V(y) d y
$$

from which (1.2) follows. 


\section{A.2 Reflectionless Potentials}

We demonstrate in the following the positivity of the Fredholm determinant of the GLM equation (2.6) in the case of reflectionless potentials.

For reflectionless potentials, where $r(k) \equiv 0, N(y, z)=-\sum_{l=1}^{N} c_{l}^{2} e^{-\kappa_{l}(y+z)}$ in $(2.8)$ is a degenerate kernel of finite rank $N$. Thus, the integral equation degenerates into a system of linear equations, and the Fredholm determinant becomes an $N$ dimensional determinant. For this reason, reflectionless potentials are so easy to treat within the formalism of IST.

The Fredholm determinant (at $\lambda=1$ ) is given by $\left(c_{1} \cdots c_{N}\right)^{2} \operatorname{det} A$, where

$$
A_{m n}=\delta_{m n}+\frac{c_{m} c_{n}}{\kappa_{n}+\kappa_{m}} e^{-\left(\kappa_{n}+\kappa_{m}\right) x}
$$

This matrix is a member of a family of matrices of the general form

$$
A_{m n}^{(\nu)}=\delta_{m n}+\frac{v_{m} v_{n}}{\left(\kappa_{n}+\kappa_{m}\right)^{\nu}}
$$

where $v_{n}$ are the components of a real vector and $\nu$ a real number. (Recall also that all $\kappa_{n}>0$.) The matrix (A.10) corresponds, of course to $v_{n}=c_{n} e^{-\kappa_{n} x}$ and $\nu=1$. It is easy to demonstrate that (A.11) is a positive definite matrix for any $\nu \geq 0$. To prove this, it is enough to verify that $\xi^{T} A \xi>0$ for any real vector $\xi$. Thus, consider

$$
\begin{aligned}
\xi^{T} A \xi & =\xi^{T} \xi+\sum_{n, m} \frac{\xi_{n} v_{n} \xi_{m} v_{m}}{\left(\kappa_{n}+\kappa_{m}\right)^{\nu}} \\
& =\xi^{T} \xi+\frac{1}{\Gamma(\nu)} \int_{0}^{\infty} s^{\nu-1}\left(\sum_{n=1}^{N} \xi_{n} v_{n} e^{-\kappa_{n} s}\right)^{2} d s \geq 0
\end{aligned}
$$

manifestly. Thus, in particular, (A.10) is positive definite and so is the corresponding Fredholm determinant.

\section{References}

[1] V.E. Zakharov and L.D. Faddeev, Funk. Analiz. Priloz. 5, 18 (1971) (in Russian), translated in Funct. Anal. Appl. 5, 280 (1971).

See also [4] below. 
[2] For a review of reflectionless potentials see e.g.,

H. B. Thacker, C. Quigg and J. L. Rosner, Phys. Rev. D 18, 274 (1978);

J. Feinberg, Ann. Phys. 309, 166 (2004) - Appendix B (and references therein).

[3] F. Cooper, A. Khare and U. Sukhatme, Phys. Rep. 251, 267 (1995); Supersymmetry in Quantum Mechanics (World Scientific, Singapore, 2001).

[4] L.D. Faddeev and L.A. Takhtajan, Hamiltonian Methods in the Theory of Solitons (Springer Verlag, Berlin, 1987).

[5] L.D. Faddeev, Usp. Mat. Nauk, 14 (1959) 57 (in Russian). Translated in the J. Math. Phys. 4 (1963) 72.

[6] L.D. Faddeev, J. Sov. Math. 5 (1976) 334. This paper is reprinted in L.D. Faddeev, 40 Years in Mathematical Physics (World Scientific, Singapore, 1995).

[7] S. Novikov, S.V. Manakov, L.P. Pitaevsky and V.E. Zakharov, Theory of Solitons

- The Inverse Scattering Method (Consultants Bureau, New York, 1984)

( Contemporary Soviet Mathematics). 